\title{
Continuous time contests with private information
}

Citation for published version (APA):

Seel, C., \& Strack, P. (2016). Continuous time contests with private information. Mathematics of Operations Research, 41(3), 1093-1107. https://doi.org/10.1287/moor.2015.0769

Document status and date:

Published: 01/08/2016

DOI:

10.1287/moor.2015.0769

Document Version:

Accepted author manuscript (Peer reviewed / editorial board version)

\section{Please check the document version of this publication:}

- A submitted manuscript is the version of the article upon submission and before peer-review. There can be important differences between the submitted version and the official published version of record.

People interested in the research are advised to contact the author for the final version of the publication, or visit the DOI to the publisher's website.

- The final author version and the galley proof are versions of the publication after peer review.

- The final published version features the final layout of the paper including the volume, issue and page numbers.

Link to publication

\footnotetext{
General rights rights.

- You may freely distribute the URL identifying the publication in the public portal. please follow below link for the End User Agreement:

www.umlib.nl/taverne-license

Take down policy

If you believe that this document breaches copyright please contact us at:

repository@maastrichtuniversity.nl

providing details and we will investigate your claim.
}

Copyright and moral rights for the publications made accessible in the public portal are retained by the authors and/or other copyright owners and it is a condition of accessing publications that users recognise and abide by the legal requirements associated with these

- Users may download and print one copy of any publication from the public portal for the purpose of private study or research.

- You may not further distribute the material or use it for any profit-making activity or commercial gain

If the publication is distributed under the terms of Article $25 \mathrm{fa}$ of the Dutch Copyright Act, indicated by the "Taverne" license above, 


\title{
CONTINUOUS TIME CONTESTS WITH PRIVATE INFORMATION
}

\author{
Christian Seel* And Philipp Strack ${ }^{\dagger}$
}

This paper introduces a class of contest models in which each player decides when to stop a privately observed Brownian motion with drift and incurs costs depending on his stopping time. The player who stops his process at the highest value wins a prize.

We prove existence and uniqueness of a Nash equilibrium outcome and derive the equilibrium distribution in closed form. As the variance tends to zero, the equilibrium outcome converges to the symmetric equilibrium of an all-pay auction. For two players and constant costs, each player's equilibrium profit decreases if the drift increases, the variance decreases, or the costs decrease.

KEYWORDS: Contests, all-pay contests, silent timing games.

\section{INTRODUCTION}

There is a large economic literature on contests. Most of this literature analyzes static models (e.g., Tullock, 1980, Lazear and Rosen, 1981, Hillman and Samet, 1987, and Siegel, 2009, 2010). Most real-world contests, however, are conducted over time rather than being a one-shot interaction; for example, think about procurement contests, job promotion contests, political campaigns, or sports leagues. Thus, there are a few more recent papers which include the dynamic aspects of a contest in their model.

A dynamic contest model requires a choice of the informational assumptions. For instance, Harris and Vickers (1987) and Moscarini and Smith (2007) analyze stochastic and dynamic two-player contests with complete information. They characterize Markov perfect equilibria and find that the effort of the leader increases in his lead up to some threshold above which the laggard resigns. More general results on the existence of Markov perfect $\epsilon$-equilibria in two-player stopping games with complete information are provided in Laraki et al. (2005) and Laraki and Solan (2013).

On the other hand, there is a literature which assumes that contestants cannot observe each other, so-called silent timing games; see, e.g., Karlin (1953) and Park and Smith (2008). In this literature, contestants can choose their stopping time, but they do not receive any new private information about their own process over time.

This paper introduces a new class of contest models without observability in which each player's strategies depend on their private information. If there is no uncertainty about the other players' progress, i.e., no private information, our model reduces to a silent

\footnotetext{
*Maastricht University, School of Business and Economics, The Netherlands, E-mail: c.seel@maastrichtuniversity.nl

${ }^{\dagger}$ Corresponding Author at University of California, Berkeley, United States, E-mail: philipp.strack@gmail.com
} 
timing game with one prize for the player who stops latest. Thus, this paper introduces private information into silent timing games.

Formally, we analyze an $n$-player contest in which each player decides when to stop a privately observed Brownian motion with drift. A strategy of a player is a bounded stopping time, i.e., the player has to stop almost surely before some deadline. As long as a player does not stop the process, he incurs flow costs of continuation. The player who stops his process at the highest value wins a prize.

The analysis proceeds as follows. Proposition 1 and Proposition 2 establish existence and uniqueness of the equilibrium distribution of the value of the stopped process. To do so, we first characterize the candidate equilibrium distribution uniquely up to its endpoints. In a second step, we use a result on Skorokhod embeddings to show that there exists a stopping strategy which leads to the candidate distribution.

In the paper, we do not fix the deadline exogenously, but the game ends once all players have stopped their processes. The equilibrium which we construct, however, remains an equilibrium if the contest ends at a sufficiently late, but fixed and predetermined deadline. Thus, we could use the more standard economic interpretation of a fixed deadline at which the results are evaluated if the deadline is sufficiently long. The bounded time restriction complicates the proof, since it drastically reduces the set of admissible stopping times.

By Proposition 3, an equilibrium stopping strategy has a simple structure, i.e., it is the first leaving time of a set. In economic terms, an agent stops whenever he either gets sufficiently optimistic or sufficiently pessimistic about his prospects in the contest, where the optimism and pessimism thresholds depend on time.

Having solved the model, we proceed by analyzing the shape of the equilibrium distribution. As uncertainty vanishes, the distribution converges to the symmetric equilibrium distribution of an all-pay auction by Proposition 4. On the one hand, the model offers a microfoundation for the use of all-pay auctions to scrutinize environments containing little uncertainty; on the other hand, it gives an equilibrium selection criterion for the equilibria of the symmetric all-pay auction analyzed in Baye et al. (1996). Furthermore, this result serves as a benchmark to discuss how our predictions differ from all-pay models if volatility is strictly positive.

Most of the related literature focuses exclusively on two players and fixed cost functions. In the last part of the paper, we also restrict attention to two players and constant costs. This allows us to derive a tractable closed-form solution for the profits of each player. More precisely, by Proposition 6, profits increase as costs increase, volatility increases, or drift decreases. Hence, contestants prefer worse technologies for both players.

This economically novel comparative statics result has an intuitive explanation: an increase in the drift or a decrease in the variance of each player makes patience a more important factor for contest success compared to chance. Hence, in equilibrium, expected 
stopping times and expected costs increase. Since the equilibrium is symmetric, the winning probability of each player remains constant. Summing up, we obtain lower expected profits.

Our setting fits applications such as procurement contests or job promotion contests. In these applications, players choose their effort levels over time. The stopping decision measures the effort, where a later stopping time should be interpreted as a larger period of effort or a higher intensity of effort. Hence, in the model, a later stopping time also induces higher costs. Moreover, we assume that - on average - effort increases output. In the model, the average productivity is measured by the drift, i.e., the average rate at which the output increases.

In most procurement contests and some job promotion contests, the research is conducted in secrecy. Thus, contestants can only observe their own progress over time which is consistent with our model description. On the other hand, a model with complete information provides a better fit for applications such as sports leagues or political campaigns (if polls are available).

\section{Related Literature}

Seel and Strack (2013) study the effect of relative performance pay on risk-taking behavior in financial markets. More precisely, fund managers decide when to stop investing in a risky asset. The manager who generates the highest profit gets a bonus. Each manager faces a liquidity constraint (maximal amount which they can loose) which drives the equilibrium construction. Seel and Strack (2013) construct an equilibrium in which managers invest in the risky asset although it has a negative expectation.

To focus on risk-taking behavior, Seel and Strack (2013) and the subsequent finance literature (Feng and Hobson, 2014a b) abstract from effort costs, which is the main decision variable in the classical contest literature. In contrast, all results in the present paper are driven by the trade-off between a higher expected cost and a higher probability of winning the contest.

Similar technical problems in characterizing the stopping region arise in Hopenhayn and Squintani (2011) who introduce private information into preemption games. They characterize the equilibrium as a solution to a free boundary problem. The solution method we develop is based on the Skorokhod embedding theorem. It provides a new approach to analyze timing games and, possibly, other stochastic games with private information. Moreover, as we show in an extension, the construction is not restricted to Brownian motion with drift, but can also be applied to other stochastic processes.

We proceed as follows. Section 2 sets up the model. In Section 3, we prove that an equilibrium exists and characterize the form of the equilibrium distribution uniquely. 
Section 4 discusses the relation to all-pay auctions and derives the main comparative statics results. Section 5 concludes. All proofs are relegated to Appendix B.

\section{THE MODEL}

There are $n \geq 2$ agents indexed by $i \in\{1,2, \ldots, n\}=N$ who face a stopping problem in continuous time. At each point in time $t \in \mathbb{R}_{+}$, agent $i$ privately observes the realization of a stochastic process $\left(X_{t}^{i}\right)_{t \in \mathbb{R}_{+}}$with

$$
X_{t}^{i}=\mu t+\sigma B_{t}^{i}
$$

The process $X^{i}$ measures the (research) progress of a player who exerts effort. It consists of a deterministic and a stochastic component: the drift $\mu \in \mathbb{R}_{++}$is the common expected change of each process $X_{t}^{i}$ per time, i.e., $\mathbb{E}\left(X_{t+\Delta}^{i}-X_{t}^{i}\right)=\mu \Delta$. The random terms $\sigma B_{t}^{i}$ are independent Brownian motions $\left(B_{t}^{i}\right)_{i \in N}$ scaled by $\sigma \in \mathbb{R}_{++}$. Note that the starting value $X_{0}^{i}$ is the same for each player and, without loss of generality, normalized to zero.

\subsection{Strategies}

A pure strategy of player $i$ is a stopping time $\tau^{i}$. As each player only observes his own process, the decision whether to stop at time $t$ only depends on the past realizations of his process $\left(X_{s}^{i}\right)_{s \leq t}$. Mathematically, the agents' stopping decision until time $t$ has to be $\mathcal{F}_{t}^{i}$-measurable, where $\mathcal{F}_{t}^{i}=\sigma\left(\left\{X_{s}^{i}: s<t\right\}\right)$ is the sigma algebra induced by the possible observations of the process $X_{s}^{i}$ before time $t$.

We require stopping times to be bounded by a real number $T<\infty$ such that $\tau^{i}<T$ almost surely. ${ }^{1}$ Note that we do not fix the value of the deadline in the description of the model, but only require the deadline to be finite. ${ }^{2}$

Although the unique equilibrium outcome of this model can be obtained in pure strategies, we incorporate mixing to make the results more general. To do so, we allow for random stopping decisions. More precisely, each agent $i$ can choose an $\left(\mathcal{F}_{t}^{i}\right)$-adapted right-continuous increasing process $\left(\kappa_{t}^{i}\right)_{t \in \mathbb{R}_{+}}, \kappa_{t}^{i} \in[0,1]$ such that, for every $t, \mathbb{P}\left(\tau^{i} \leq\right.$ $\left.t \mid \mathcal{F}_{t}^{i}\right)=\kappa_{t}^{i}$. A pure strategy is equivalent to a process $\kappa_{t}^{i}$ that equals zero for all $t<\tau$ and one otherwise. ${ }^{3}$

\footnotetext{
${ }^{1}$ The equilibrium we derive in the paper remains an equilibrium if we do not restrict agents to bounded stopping times, but only require stopping times to be finite, i.e., $\tau<\infty$.

${ }^{2}$ In the equilibrium which we construct based on Proposition 3 , however, all players stop before a fixed time $T$. Since a fixed deadline reduces the strategy space, any equilibrium which we construct remains an equilibrium for a model with a fixed deadline which is longer than $T$ (for details, see discussion after Proposition 3).

${ }^{3}$ Touzi and Vieille (2002) introduce the same definition of strategies for Dynkin games. In that setting, it is equivalent to (i) mixed strategies in the sense of Aumann (1961) and (ii) behaviorally mixed strategies as defined for continuous time stopping games in Bismut (1979). Shmaya and Solan (2014) show that
} 


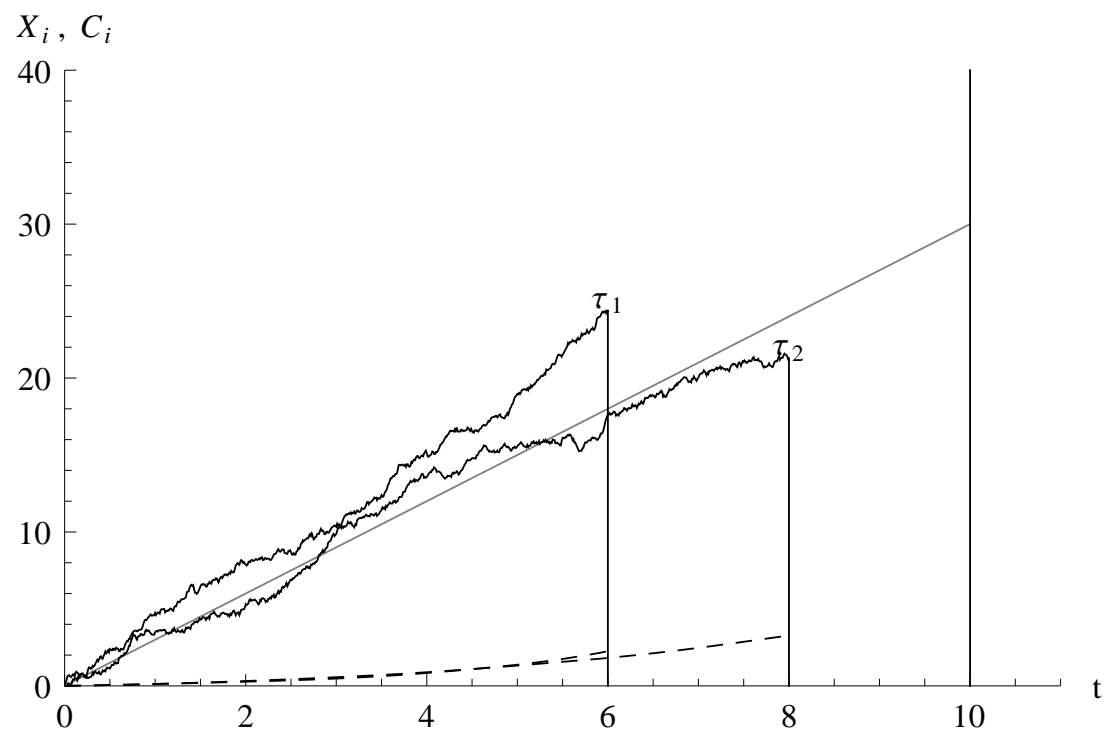

Figure 1.- An example for the game with two agents $i \in\{1,2\}$. Time is depicted on the $x$-axis, while the value $X_{t}^{i}$ (solid line) and total costs (dotted line) are depicted on the $y$-axis. The parameters are $c\left(X_{t}^{i}\right)=\frac{1}{10} \int_{0}^{t} \exp \left(\frac{1}{10} X_{s}^{i}\right) \mathrm{d} s, \mu=3, \sigma=1, T=10$.

\subsection{Payoffs}

The player who stops his process at the highest value wins a prize; ties are broken randomly. ${ }^{4}$ Until he stops, each player incurs flow costs $c: \mathbb{R} \rightarrow \mathbb{R}_{++}$which depend on the current value of the process $X_{t}^{i}$, but not on the time $t$. We normalize the prize to one, since agents only care about the trade-off between winning probability and cost-prize ratio. The payoff $\Pi^{i}$ is thus

$$
\Pi^{i}=\frac{1}{k} \mathbf{1}_{\left\{X_{\tau^{i}}^{i}=\max _{j \in N} X_{\tau^{j}}^{j}\right\}}-\int_{0}^{\tau^{i}} c\left(X_{t}^{i}\right) \mathrm{d} t,
$$

where $k=\left|\left\{i \in N: X_{\tau^{i}}^{i}=\max _{j \in N} X_{\tau^{j}}^{j}\right\}\right|$ is the number of agents who stop at the highest value. All agents maximize their expected profit $\pi^{i}=\mathbb{E}\left(\Pi^{i}\right)$. The cost function satisfies the following mild assumption:

Assumption 1 The cost function $c: \mathbb{R} \rightarrow \mathbb{R}_{++}$is continuous, positive, and bounded away from zero.

Figure 1 illustrates processes, stopping decisions, and corresponding costs in an example. Realizations of the processes until the stopping time are depicted in solid lines, while

under weak conditions, all types of random stopping times are equivalent. For an analogous discussion in discrete time stopping games, see Solan et al. (2012).

${ }^{4}$ The assumption of a uniform tie-breaking rule is irrelevant, since the equilibrium distributions have no atoms, i.e., the probability of a tie is zero. 
the corresponding total costs are depicted in dotted lines. In the example, player 1 stops before player 2 stops. Nonetheless, player 1 receives the prize, since she stops her process at a higher value than player 2 .

\subsection{Main Characterization Results}

In the next section, we derive several results about the equilibrium set of the game. They can be summarized as follows:

THEOREM 1 The game has a pure strategy Nash equilibrium. There exists a unique distribution $F$ over stopped values such that in any Nash equilibrium, all players choose strategies inducing the distribution $F$. The equilibrium payoffs are uniquely determined.

\section{EQUILIBRIUM CONSTRUCTION}

In this section, we first establish some necessary conditions on the distribution functions of the stopped processes in equilibrium. In a second step, we prove existence and uniqueness of the Nash equilibrium outcome and determine the equilibrium distributions depending on the cost function.

Every strategy of agent $i$ induces a (potentially non-smooth) cumulative distribution function (cdf) $F^{i}: \mathbb{R} \rightarrow[0,1]$ of his stopped process $F^{i}(x)=\mathbb{P}\left(X_{\tau^{i}}^{i} \leq x\right)$. Intuitively, $F^{i}(x)$ is the probability that a player stops with an output less or equal to $x$.

Denote the endpoints of the support of the equilibrium distribution of player $i$ by

$$
\begin{aligned}
& \underline{x}^{i}=\inf \left\{x: F^{i}(x)>0\right\} \\
& \bar{x}^{i}=\sup \left\{x: F^{i}(x)<1\right\} .
\end{aligned}
$$

Let $\underline{x}=\max _{i \in N} \underline{x}^{i}$ and $\bar{x}=\max _{i \in N} \bar{x}^{i}$. In the next step, we establish a series of auxiliary results that are crucial to prove uniqueness of the equilibrium distribution.

LEMma 1 No player places a mass point in the interior of the state space, i.e., for all $i$, for all $x>\underline{x}: \mathbb{P}\left(X_{\tau^{i}}^{i}=x\right)=0$. At least one player has no mass at the left endpoint, i.e., $F^{i}(\underline{x})=0$, for at least one player $i$.

We omit the proof of Lemma 1, since it is simply a specialization of the standard logic in static game theory with a continuous state space; see, e.g., Burdett and Judd (1983). Intuitively, in equilibrium, no player can place a mass point in the interior of the state space, since no other player would then stop slightly below the mass point.

Since $F^{i}(\underline{x})=0$ for at least one player $i$, we have $\mathbb{P}\left(\max _{i} X_{\tau^{i}}^{i}>\underline{x}\right)=1$. Moreover, as no player sets a mass point inside the support, we have $\mathbb{P}\left(X_{\tau^{j}}^{j}=X_{\tau^{i}}^{i} \mid X_{\tau^{i}}^{i}>\underline{x}\right)=0$. Thus, the probability of a tie is equal to zero. 
Thus, we can express the winning probability of player $i$ if he stops at $X_{\tau^{i}}^{i}=x$, given the distributions of the other players, as

$$
w^{i}(x)=\mathbb{P}\left(\max _{j \neq i} X_{\tau^{j}}^{j} \leq x\right)=\prod_{j \neq i} F^{j}(x)
$$

Lemma 2 At least two players stop with positive probability on every interval $I=(a, b) \subset[\underline{x}, \bar{x}]$ (the identity of the players who stop may depend on the interval).

Lemma 3 All players have the same equilibrium distribution function, $F^{i}=F$, for all $i \in N$.

Lemma 2 and 3 are the crucial steps for the uniqueness proof. In particular, Lemma 2 does not hold in static auction theory. Intuitively, the support of an equilibrium bid distribution in an auction is not necessary connected; see, for instance, Baye et al. (1996), Theorem 1, who construct an equilibrium in which a player places a mass point at zero and randomizes his bid on some interval $[x, \bar{x}]$. In our case, every bounded time stopping strategy induces a distribution of the stopped process with a connected support. This property of bounded stopping strategies greatly facilitates the uniqueness proofs.

The following lemma is an immediate consequence from the symmetry of the equilibrium distribution (Lemma 3 ) and the fact that for at least one agent $w^{i}(\underline{x})=0$ (Lemma 1$)$.

Lemma 4 Each player loses with certainty at $\underline{x}$, i.e., $w^{i}(\underline{x})=0$ for all $i \in N$.

As players have symmetric distributions, we henceforth drop the superscript $i$. The next result uses Itô's lemma to determine the ordinary differential equation (ODE) for the winning probability $w$ :

LEMma 5 For all $x \in(\underline{x}, \bar{x})$, the winning probability is twice differentiable and satisfies

$$
c(x)=\mu w^{\prime}(x)+\frac{\sigma^{2}}{2} w^{\prime \prime}(x) .
$$

Intuitively, in equilibrium the expected costs from continuation (lhs of Eq. (1)) must equal the expected gain in winning probability (rhs of Eq. (1)) and the agent is indifferent between stopping and continuing at every point of the support.

As Eq. (1) is a second order ODE, we use two boundary conditions to determine $w$ uniquely. One boundary condition is $w(\underline{x})=0$ from Lemma 4 . We determine the other one in the following lemma:

LEMMA $6 \quad$ In equilibrium, $w^{\prime}(\underline{x})=0$. 
Intuitively, Lemma 6 imposes a smooth-pasting condition which ensures that players have no incentive to continue at the left endpoint of their support.

Define $\phi(x)=\exp \left(\frac{-2 \mu x}{\sigma^{2}}\right)$. To calculate the solution to Eq. (1), note that $b_{1}+b_{2} \phi(x)$ (for any $\left.b_{1}, b_{2} \in \mathbb{R}\right)$ is the general solution to the homogeneous equation $0=\mu w^{\prime}(x)+\frac{\sigma^{2}}{2} w^{\prime \prime}(x)$. To solve the inhomogeneous equation, we apply the variation-of-constants formula. We then use the two boundary conditions to calculate the unique candidate solution. Finally, we rearrange with Fubini's Theorem to get

$$
w(x)= \begin{cases}0 & \text { for } x<\underline{x} \\ \frac{1}{\mu} \int_{\underline{x}}^{x} c(z)(1-\phi(x-z)) \mathrm{d} z & \text { for } x \in[\underline{x}, \bar{x}] \\ 1 & \text { for } \bar{x}<x .\end{cases}
$$

By symmetry of the equilibrium strategy, the equilibrium distribution satisfies $F(x)=$ $\sqrt[n-1]{w(x)}$. Consequently, any equilibrium distribution must be of the form

$$
G(x ; \underline{x})= \begin{cases}0 & \text { for all } x \leq \underline{x} \\ \min \left\{1, \sqrt[n-1]{\frac{1}{\mu} \int_{\underline{x}}^{x} c(z)(1-\phi(x-z)) \mathrm{d} z}\right\} & \text { for all } x>\underline{x} .\end{cases}
$$

In the next step, we verify that $G$ is an absolutely continuous cumulative distribution function.

Lemma 7 For every left endpoint $\underline{x}$, the function $G$ defined in Eq. (2) is absolutely continuous, nondecreasing and $\lim _{x \rightarrow \infty} G(x ; \underline{x})=1$.

Note that Eq. (2) describes the equilibrium distribution only up to the left endpoint of the support. To determine the left endpoint of the support, we use the following condition which is necessary for a distribution $G$ to be the outcome of a stopping strategy $\tau$.

LEMma 8 If $\tau \leq T<\infty$ is a bounded stopping time that induces the absolutely continuous distribution $H$, i.e., $H(z)=\mathbb{P}\left(X_{\tau} \leq z\right)$, then $1=\int_{\underline{x}}^{\bar{x}} \phi(x) H^{\prime}(x) \mathrm{d} x$.

Lemma 8 and Eq. (2) imply that the left endpoint $\underline{x}$ must solve

$$
1=\int_{\mathbb{R}} \phi(x) G^{\prime}(x ; \underline{x}) \mathrm{d} x .
$$

The next result shows that there is unique endpoint $\underline{x}$ solving Eq. (3). Furthermore, it establishes that every profile in which all players use a strategy which induces the distribution $G(x ; \underline{x})$ from Eq. (2) with the unique $\underline{x}$ is a Nash equilibrium.

Proposition 1 There exists a unique $\underline{x}$ such that a strategy profile is a Nash equilibrium if and only if each player's strategy induces the distribution $F(x)=G(x ; \underline{x})$ defined in Eq. (2). 
To build up more intuition for Proposition 1, recall that ties occur with probability zero and that equilibrium distributions are symmetric. Moreover, note that an equilibrium strategy of a player needs to be an optimal stopping time for this problem and not necessarily the minimal optimal stopping as in many finance problems. Since no new information about the other players arrives over time and the best-response problem is Markovian, the optimal stopping decision does not depend on time, but only on the value $X_{t}$.

Our construction of the distribution $F$ ensures that if all agents induce $F$, the remaining agent is indifferent between all stopping strategies which stop before the process leaves the support $[\underline{x}, \bar{x}]$ the first time. As every agent wins with probability one at the right endpoint $\bar{x}$, it is strictly optimal to stop there. The smooth pasting condition $w^{\prime}(\underline{x})=0$ imposed by Lemma 6 ensures that it is also optimal to stop at the left endpoint. Thus, if all players induce $F$, we have a Nash equilibrium, since each agent chooses a best response when he stops with probability 1 in $[\underline{x}, \bar{x}]$.

Furthermore, since the optimal stopping decision does not depend on time, the same logic holds after every (private) history. Thus, any strategy profile in which all players induce the distribution $F$ and stop immediately whenever $X_{t} \notin(\underline{x}, \bar{x})$ also satisfies Nash refinements such as sequential rationality, time-consistency, and subgame-perfection.

So far, we have verified that a bounded stopping time $\tau \leq T<\infty$ is an equilibrium strategy if and only if it induces the distribution $F$, i.e., $F(z)=\mathbb{P}\left(X_{\tau} \leq z\right)$. To show that the game has a Nash equilibrium, the existence of a bounded stopping time inducing $F$ remains to be established. The problem of finding a stopping time $\tau$ such that a Brownian motion stopped at $\tau$ has a given centered probability distribution $F$, i.e., $F \sim B_{\tau}$, is known in the probability literature as the Skorokhod embedding problem (SEP). Since its initial formulation in Skorokhod $(1961,1965)$, many solutions have been derived; for a survey article, see Oblój (2004). The proof of the following proposition verifies a sufficient condition for a bounded time embedding from a recent mathematical paper.

Proposition 2 The game has a Nash equilibrium. In equilibrium, the strategy of each player induces the distribution $F$ defined in Eq. (2).

The uniqueness result differs from related models such as symmetric all-pay auctions or symmetric silent timing games, in which there are usually multiple equilibria with different equilibrium distributions. In some of these equilibria only a subset of players is active, i.e., submits a positive bid in the auction or does not stop directly in the silent timing game. Here, the variance leads all players to be active in the game. Hence, we obtain a unique equilibrium distribution.

A conceptual improvement on the literature is the bounded time requirement $\tau<T$, which makes the equilibrium derivation applicable to contests with a fixed deadline. To 
see that this result is not trivial to obtain, note that for any fixed time horizon $T$, there exists a positive probability that $X_{t}$ does not leave any interval $[a, b]$ with $a<X_{0}<b$. Hence, a simple construction through a mixture over "tunnel" strategies of the form

$$
\tau_{a, b}=\inf \left\{t \in \mathbb{R}_{+}: X_{t} \notin[a, b]\right\}
$$

i.e., first leaving times of an interval, cannot be used to implement $F$.

In the following, we show that a different simple strategy induces the equilibrium distribution. For this purpose, we define:

Definition 1 (Barrier Stopping Time) A stopping time $\tau$ is a barrier stopping time if it is of the form

$$
\tau=\inf \left\{t \in \mathbb{R}_{+}:\left(t, X_{t}\right) \in C\right\}
$$

where $C \subset \mathbb{R}_{+} \times \mathbb{R}$ is a set such that $(t, x) \in C$ implies $\left(t^{\prime}, x\right) \in C$ if $t^{\prime}>t$.

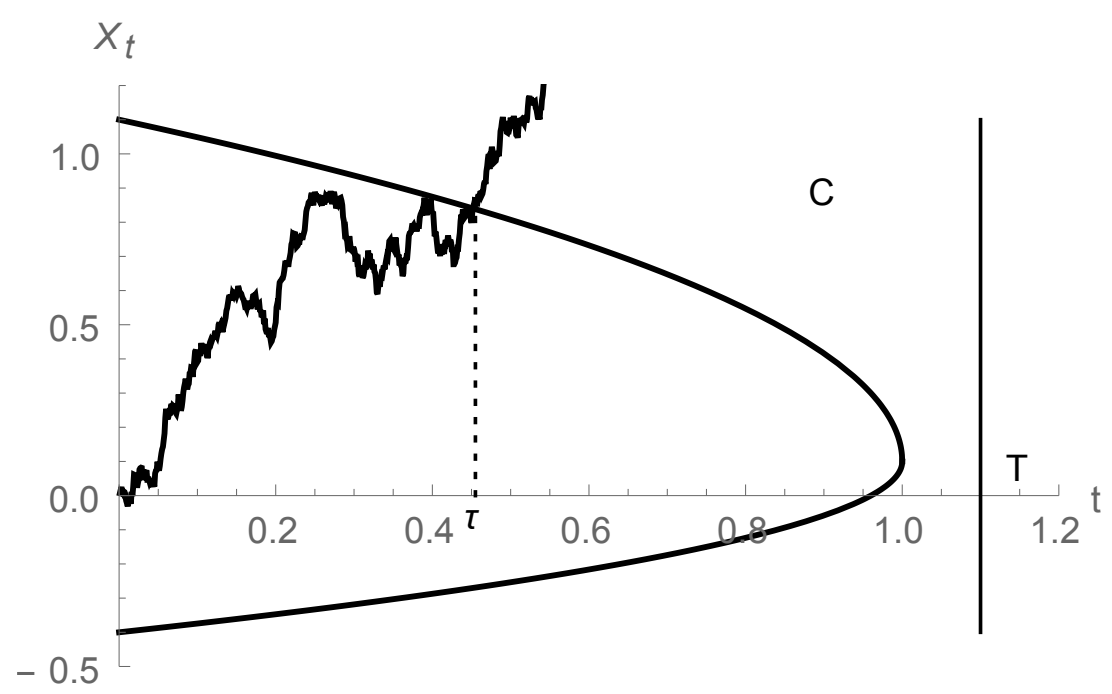

FiguRE 2.- An example of a barrier stopping time.

Barrier stopping times are relatively simple, as they only condition on the value of the process and the time, but not on the history.

Proposition 3 There exists a bounded equilibrium stopping time which is a barrier stopping time.

An equilibrium in which each player uses a barrier stopping time has a nice economic intuition: whenever a player gets sufficiently optimistic or sufficiently pessimistic about 
her prospects in the contest, she stops to exert effort. In Figure 2, we draw a sample path of the process and a barrier stopping time. The agent is sufficiently optimistic if she hits the upper threshold, while she is sufficiently pessimistic if she hits the lower threshold. Figure 2 also illustrates our result for contests with a fixed deadline: a barrier stopping time which induces the distribution defined in Eq. (2) stops almost surely before some finite point in time. In a contest with a fixed, finite deadline which is greater or equal to this point in time, each player can thus induce the distribution defined in Eq. (2). Since the strategy space is restricted by the fixed deadline, the equilibrium derived for the larger strategy space without a fixed deadline remains an equilibrium in the game with a fixed, but sufficiently long deadline. For given parameters, it is possible to calculate an upper bound for the critical contest length using Lemma 12 in Appendix A.

\section{EQUILIBRIUM ANALYSIS}

\subsection{Convergence to the All-pay Auction}

This subsection considers the relationship between the literature on all-pay auctions and our model for vanishing noise. We first establish an auxiliary result about the left endpoint:

LEMMA 9 If the noise vanishes, the left endpoint of the equilibrium distribution defined in Eq. (2) converges to zero, i.e., $\lim _{\sigma \rightarrow 0} \underline{x}=0$.

Taking the limit $\sigma \rightarrow 0$, the equilibrium distribution converges to

$$
\lim _{\sigma \rightarrow 0} F(x)=\sqrt[n-1]{\frac{1}{\mu} \int_{0}^{x} c(z) \mathrm{d} z} .
$$

In a static $n$-player all-pay auction, the symmetric equilibrium distribution is

$$
F(x)=\sqrt[n-1]{\frac{x}{v}}
$$

where $x$ is the total outlay of a participant and $v$ is her valuation; see, e.g., Hillman and Samet (1987). In our case, the total outlay depends on the flow costs at each point, the speed of research $\mu$, and the stopping time $\tau$. More precisely, it is $\int_{0}^{x} \frac{c(z)}{\mu} \mathrm{d} z$. The valuation $v$ in the analysis of Hillman and Samet (1987) coincides with the prize $p$-which we have normalized to one - in our contest. This yields the following proposition:

Proposition 4 For vanishing noise, the equilibrium distribution converges to the symmetric equilibrium distribution of an all-pay auction with homogeneous valuations. 
Thus, our model supports the use of all-pay auctions to analyze contests in which variance is negligible. Moreover, the symmetric all-pay auction has multiple equilibria - for a full characterization see Baye et al. (1996), Theorem 1. This paper offers an equilibrium selection criterion in favor of the symmetric equilibrium. Intuitively, all other equilibria of the symmetric all-pay auction include mass points at zero for some players, which is not possible in our model for any positive $\sigma$ by Lemma 4 .

\subsection{Comparative Statics and Rent Dispersion}

Proposition 4 has linked all-pay auctions with complete information to our model for the case of vanishing noise. In the following, we scrutinize how predictions differ for positive noise. In a symmetric all-pay auction with complete information, agents make zero profits in equilibrium. This does not hold true in our model for any positive level of variance $\sigma$ :

Proposition 5 In equilibrium, all agents make strictly positive expected profits.

Intuitively, agents generate informational rents through the private information about their research progress. A similar result is known in the literature on all-pay auctions with incomplete information, see, e.g., Hillman and Riley (1989), Amann and Leininger (1996), Krishna and Morgan (1997), and Moldovanu and Sela (2001). In these models, each participant takes a draw from a common distribution. The outcome of each draw is private information and determines the effort cost or valuation. In contrast to this, private information about one's progress arrives continuously over time in our model.

\subsection{Two Players and Constant Costs}

We henceforth restrict attention to the case $n=2$ and $c(x)=c$ to get more explicit results. We require some additional notation. In particular, we denote the principal branch of the Lambert $W$-function by $W_{0}:\left[-\frac{1}{e}, \infty\right) \rightarrow \mathbb{R}_{+}$. This branch is implicitly defined on $\left[-\frac{1}{e}, \infty\right)$ as the unique solution of $x=W(x) \exp (W(x)), W \geq-1$. Moreover, define $h: \mathbb{R}_{+} \rightarrow[0,1]$ by

$$
h(y)=\exp \left(-y-1-W_{0}(-\exp (-1-y))\right)
$$

The next lemma pins down the left and right endpoints of the support. 


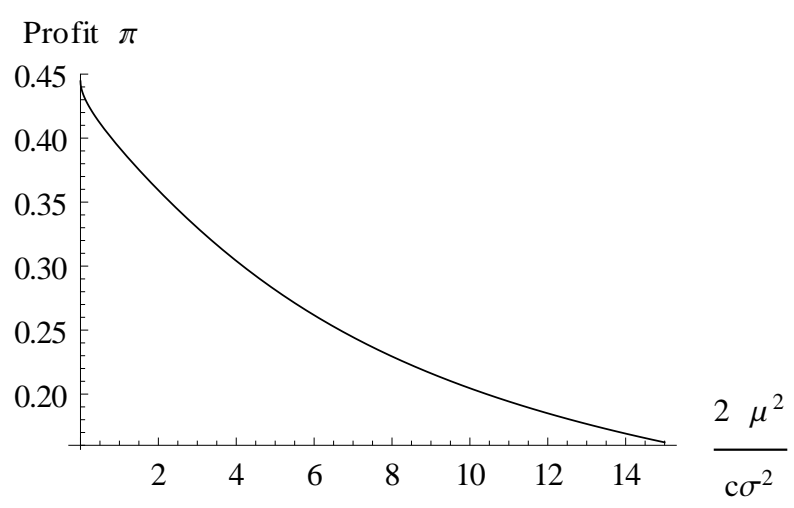

FiguRE 3.- This figure shows the equilibrium profit $\pi$ of the agents on the $y$-axis for $n=2$ and constant cost-functions $c(x)=c \in \mathbb{R}_{++}$with $y=\frac{2 \mu^{2}}{c \sigma^{2}}$ on the $x$-axis.

LEMMA 10 The left and right endpoints are

$$
\begin{aligned}
& \underline{x}=\frac{\sigma^{2}}{2 \mu}\left(2 \log \left(1-h\left(\frac{2 \mu^{2}}{c \sigma^{2}}\right)\right)-\log \left(\frac{4 \mu^{2}}{c \sigma^{2}}\right)\right) \\
& \bar{x}=\frac{\sigma^{2}}{2 \mu}\left(2 \log \left(1-h\left(\frac{2 \mu^{2}}{c \sigma^{2}}\right)\right)-\log \left(\frac{4 \mu^{2}}{c \sigma^{2}}\right)-\log \left(h\left(\frac{2 \mu^{2}}{c \sigma^{2}}\right)\right)\right) .
\end{aligned}
$$

The next lemma derives a closed-form solution of the profits $\pi$ of each player.

LEMMA 11 The equilibrium profit of each player depends only on the ratio $y=\frac{2 \mu^{2}}{c \sigma^{2}}$. It is given by

$$
\pi=\frac{(1-h(y))^{2}}{2 y^{2}}-\frac{2 \log (1-h(y))-\log (2 y)-1}{y} .
$$

For an illustration of the above formula, see Figure 3. The previous lemma enables us to establish the main comparative static result of this paper.

Proposition 6 The equilibrium profit of each player increases if costs increase, variance increases, or drift decreases.

To get an intuition for the result, we decompose the term $\frac{2 \mu^{2}}{c \sigma^{2}}$, which determines the equilibrium profit of the players, into two parts:

$$
\frac{2 \mu^{2}}{c \sigma^{2}}=\underbrace{\frac{\mu}{c}}_{\text {Productivity }} \times \underbrace{\frac{2 \mu}{\sigma^{2}}}_{\text {Signal to noise ratio }} .
$$

The first term $\frac{\mu}{c}$ is a deterministic measure of productivity per unit of time. On the other hand, the second term $\frac{2 \mu}{\sigma^{2}}$ measures the impact of randomness $\sigma^{2}$ on the final outcome. As the drift of both competitors increases or the variance decreases, patience becomes 
more critical to winning than chance. Hence, the expected stopping time in equilibrium increases and expected total costs increase. As each player's winning probability remains

$\frac{1}{2}$ by symmetry, profits decrease. In summary, participants prefer to have worse - more costly, more random, or less productive - technologies.

\subsection{Extension to Other Stochastic Processes}

Up to now, we have derived our results for Brownian motion with drift. However, it is straightforward to extend them to any process $Z_{t}$ such that $Z_{t}$ is the result of a strictly monotone transformation $\rho: \mathbb{R} \rightarrow \mathbb{R}$ of Brownian motion with drift $X_{t}$, i.e., $Z_{t}=\rho\left(X_{t}\right)$. First, observe that the success process $Z_{t}$ is only payoff relevant as an ordinal variable, as agent $i$ receives a prize if

$$
Z_{\tau^{i}}^{i}>\max _{j \neq i} Z_{\tau^{j}}^{j} \Leftrightarrow \rho^{-1}\left(Z_{\tau^{i}}^{i}\right)>\max _{j \neq i} \rho^{-1}\left(Z_{\tau^{j}}^{j}\right) \Leftrightarrow X_{\tau^{i}}^{i}>\max _{j \neq i} X_{\tau^{j}}^{j}
$$

As costs $c$ depend on the value of the process $Z_{t}$, we define the cost function $\tilde{c}(x)=c(\rho(x))$ for the process $X_{t}$. Let $\tilde{F}$ be the equilibrium distribution for the game with a Brownian motion with drift $X_{t}$ and cost $\tilde{c}$, i.e., $X_{\tau^{i}}^{i} \sim \tilde{F}$. The equilibrium distribution of the original game is now given by $F(x)=\tilde{F}\left(\rho^{-1}(x)\right)$, i.e., $Z_{\tau^{i}}^{i} \sim F$. For example, this generalization includes geometric Brownian motion with drift $\left(Z_{t}=\exp \left(X_{t}\right)\right)$.

\section{CONCLUSION AND DISCUSSION}

In this paper, we have introduced a new class of contest models in continuous time with private information. Under mild assumptions on the cost function, a Nash equilibrium exists. We have characterized the unique equilibrium distribution of the stopped process. Moreover, there exists a bounded stopping strategy — the first leaving time of a set — which leads to this distribution. For two players and constant costs, each player prefers mutually higher research costs, worse technologies, and higher uncertainty. This economically novel feature results from chance becoming more important for contest success than patience. From a technical perspective, we have introduced a method to construct equilibria in continuous time games. This methodological contribution should enhance future research on timing games and other stochastic games with private information.

In future research, it would be interesting to include asymmetries in the parameters (drift, variance, costs, and starting value) into the model. The main problem in this case is to characterize the appropriate boundary conditions to Eq. (1). Our proofs do not extend in a straightforward way since Lemma 4 and 6 rely on symmetry arguments. Moreover, the derivation of the equilibrium relies on the Markovian structure of each player's optimization problem. It remains an open question how to deal with non-Markovian settings. 


\section{APPENDIX A: PRELIMINARY RESULTS}

Before we prove the main results from the text in Appendix B, we introduce a technical auxiliary result from Ankirchner and Strack (2011) and a remark which turn out to be useful for the main proofs. In addition to the assumption stated in the next lemma, Ankirchner and Strack (2011) assume that the condition in Lemma 8 holds. They define $g(x)=F^{-1}(\Phi(x))$, where $\Phi(x)=\frac{1}{\sqrt{2 \pi}} \int_{-\infty}^{x} \exp \left(\frac{z^{2}}{2}\right) \mathrm{d} z$ is the density function of the normal distribution.

Lemma 12 (Ankirchner and Strack, 2011, Theorem 2) Suppose that $g$ is Lipschitzcontinuous with Lipschitz constant $\sqrt{T}$. Then the distribution $F$ can be embedded in $X_{t}=\mu t+B_{t}$, with a stopping time that stops almost surely before $T$.

Let us denote the first leaving time of the interval $[a, b]$ of agent $i$ after time $t$ by

$$
\tau_{(a, b)}^{t, i}=\inf \left\{s \geq t: X_{s}^{i} \notin(a, b)\right\}
$$

REMARK 1 The continuation strategy $\tau_{(a, b)}^{t, i}$ is strictly better than stopping at $x$ for agent i iff

$$
\mathbb{E}\left(w^{i}\left(X_{\tau_{(a, b)}^{t, i}}^{i}\right)-\int_{t}^{\tau_{(a, b)}^{t, i}} c\left(X_{s}^{i}\right) \mathrm{d} s \mid X_{t}^{i}=x\right)>w^{i}(x) .
$$

If $\tau_{(a, b)}^{t, i}$ is a profitable continuation strategy, the continuation strategy $\tau_{(a, b)}^{t, i} \wedge T$ is also profitable for a sufficiently long, but finite time horizon $T$, since

$$
\begin{aligned}
\lim _{T \rightarrow \infty} & \mathbb{E}\left(w ^ { i } \left(X_{\tau_{(a, b)}^{i}, i}^{i} \wedge T\right.\right. \\
= & \left.\int_{t}^{\tau_{(a, b)}^{t, i} \wedge T} c\left(X_{s}^{i}\right) \mathrm{d} s \mid X_{t}^{i}=x\right) \\
& \mathbb{E}\left(w^{i}\left(X_{\tau_{(a, b)}^{i, i}}^{i}\right)-\int_{t}^{\tau_{(a, b)}^{t, i}} c\left(X_{s}^{i}\right) \mathrm{d} s \mid X_{t}^{i}=x\right) .
\end{aligned}
$$

Thus, in the next proofs, it suffices to show that $\tau_{(a, b)}^{t, i}$ is a profitable deviation to rule out equilibrium candidates.

\section{APPENDIX B}

We are now ready to prove our main results:

Proof of Lemma 2: As players have to use bounded time stopping strategies, $F^{i}$ is strictly increasing on $\left[\underline{x}^{i}, \bar{x}^{i}\right]$ by Corollary 1 in Ankirchner and Strack (2011). Put differently, each player $i$ stops with positive probability on every subinterval of $\left[\underline{x}^{i}, \bar{x}^{i}\right]$. Hence, it suffices to show that at least two players have $\bar{x}$ as their right endpoint. Assume, by contradiction, only player $i$ has $\bar{x}$ as his right endpoint. Denote $\bar{x}^{-i}=\max _{j \neq i} \bar{x}^{j}$. Then, by continuity of $F^{j}$, player $i$ strictly prefers to stop at $\bar{x}^{-i}$, which yields him the 
maximal possible winning probability of 1 without any additional costs. This contradicts the optimality of a strategy, which stops at $\bar{x}^{i}>\bar{x}^{-i}$.

Q.E.D.

Proof of Lemma 3: Let us first argue that stopping is always optimal for each agent. Towards a contradiction, assume that player $i$ strictly prefers to continue at some value $x \in\left[\underline{x}^{i}, \bar{x}^{i}\right]$ and by continuity in an $\epsilon$-ball around it. Thus, at any time $t$, stopping in $(x-\epsilon, x-\epsilon)$ is worse than the continuation strategy $\tau_{(x-\epsilon, x+\epsilon)}^{t, i}$, i.e., the player never stops in $(x-\epsilon, x+\epsilon)$. However, by Corollary 1 in Ankirchner and Strack (2011), for any bounded stopping time, each player stops in every interval $I \subset\left[\underline{x}^{i}, \bar{x}^{i}\right]$ with positive probability, i.e., we have a contradiction. The rest of the proof is split in several steps.

Step 1: $F^{i}(x) \leq F^{j}(x)$ if and only if $w^{j}(x) \leq w^{i}(x)$ for all $x>\underline{x}$.

By definition, $w^{i}(x) F^{i}(x)=w^{j}(x) F^{j}(x)$. Hence, $\frac{w^{i}(x)}{w^{j}(x)}=\frac{F^{j}(x)}{F^{i}(x)}$.

Step 2: $w^{i}\left(\underline{x}^{j}\right) \geq w^{j}\left(\underline{x}^{j}\right)$ implies $F^{i}(x) \leq F^{j}(x)$ for almost all $x$.

Step 2.1: We first prove $w^{j}(x) \leq w^{i}(x)$ for all $x \in\left(\underline{x}^{j}, \bar{x}^{j}\right) \cap\left(\underline{x}^{i}, \bar{x}^{i}\right)$. For all $x \in\left(\underline{x}^{j}, \bar{x}^{j}\right) \cap$ $\left(\underline{x}^{i}, \bar{x}^{i}\right)$ player $i$ weakly prefers stopping at $x$ to the continuation strategy $\tau_{\left(\underline{x}^{j}, \bar{x}^{j}\right)}^{t, i}$

$$
\mathbb{E}\left(w^{i}\left(X_{\tau_{\left(\underline{x}^{j}, \bar{x}^{j}\right)}^{t, i}}^{i}\right)-\int_{0}^{\left.\tau^{t, i} \underline{\underline{x}}^{j}, \bar{x}^{j}\right)} c\left(X_{s}^{i}\right) \mathrm{d} s \mid X_{t}^{i}=x\right) \leq w^{i}(x) .
$$

As $\underline{x}^{j}$ and $\bar{x}^{j}$ are in the support of player $j$ the continuation strategy $\tau_{\left(\underline{x}^{j}, \bar{x}^{j}\right)}^{t, j}$ is optimal for every point $x \in\left(\underline{x}^{j}, \bar{x}^{j}\right)$, which yields

$$
\mathbb{E}\left(w^{j}\left(X_{\tau_{\left(\underline{x}^{j}, \bar{x}^{j}\right)}^{t}}^{j, j}\right)-\int_{0}^{\left.\tau^{t, j} \underline{\underline{x}}^{j}, \bar{x}^{j}\right)} c\left(X_{s}^{j}\right) \mathrm{d} s \mid X_{t}^{j}=x\right) \geq w^{j}(x) .
$$

By assumption, $w^{i}\left(\underline{x}^{j}\right) \geq w^{j}\left(\underline{x}^{j}\right)$. Moreover, $F^{i}(\bar{x}) \leq 1=F^{j}\left(\bar{x}^{j}\right)$ which yields by Step 1 $w^{i}\left(\bar{x}^{j}\right) \geq w^{j}\left(\bar{x}^{j}\right)$. Hence $w^{j}\left(X_{\tau_{(\underline{x}, \bar{x})}^{t, j}}^{j}\right) \leq w^{i}\left(X_{\tau_{\left(\underline{\left.x^{j}, \bar{x}^{j}\right)}\right.}^{i}}^{i}\right)$ and as

$$
\mathbb{E}\left(\int_{0}^{\tau_{\left(\underline{x}^{j}, \bar{x}^{j}\right)}^{t, i}} c\left(X_{s}^{i}\right) \mathrm{d} s \mid X_{t}^{i}=x\right)=\mathbb{E}\left(\int_{0}^{\tau_{\left(\underline{x}^{j}, \bar{x}^{j}\right)}^{t, j}} c\left(X_{s}^{j}\right) \mathrm{d} s \mid X_{t}^{j}=x\right)
$$

the above equations imply $w^{j}(x) \leq w^{i}(x)$ for all $x \in\left(\underline{x}^{j}, \bar{x}^{j}\right) \cap\left(\underline{x}^{i}, \bar{x}^{i}\right)$.

Step 2.2: In this step, we prove $w^{i}(x) \geq w^{j}(x)$ for all $x \geq \bar{x}^{i} \wedge \bar{x}^{j}$. By Step 2.1, $F^{i}(x) \leq F^{j}(x)$ for all $x \in\left(\underline{x}^{j}, \bar{x}^{j}\right) \cap\left(\underline{x}^{i}, \bar{x}^{i}\right)$. As $1=F^{i}\left(\bar{x}^{i}\right) \leq F^{j}\left(\bar{x}^{i}\right)$ it follows that $\bar{x}^{j} \leq \bar{x}^{i}$. As $F^{i}(x) \leq 1=F^{j}(x)$ it follows by Step 1 that $w^{j}(x) \leq w^{i}(x)$ for all $x \geq \bar{x}^{j}$. 
Step 2.3: In the final step, we prove $w^{i}\left(\underline{x}^{j}\right) \geq w^{j}\left(\underline{x}^{j}\right)$ for all $x \leq \underline{x}^{i} \vee \underline{x}^{j}$. First let $\underline{x}^{i}<\underline{x}^{j}$. As no agent places a mass point in the interior it follows that $F^{j}\left(\underline{x}^{j}\right)=0$ and thus $w^{i}\left(\underline{x}^{j}\right)=0$. Using the result of the first step it follows that for all $x \leq \underline{x}^{j}$ $w^{j}\left(\underline{x}^{j}\right) \leq \lim _{x \searrow \underline{x}^{j}} w^{j}(x) \leq \lim _{x \searrow \underline{x}^{j}} w^{i}(x)=0$. Hence, $w^{i}(x)=w^{j}(x)=0$ for all $x \leq \underline{x}^{j}$. Let $\underline{x}^{j} \leq \underline{x}^{i}$. As $F^{i}(x)=0 \leq F^{j}(x)$ for all $x<\underline{x}^{i}$ it follows from Step 1 that $w^{j}(x) \leq w^{i}(x)$ for all $x<\underline{x}^{i}$.

Hence, $w^{j}(x) \leq w^{i}(x)$ almost everywhere and by Step $1 F^{i} \leq F^{j}$ almost everywhere.

Step 3: $F^{i}=F^{j}$ for all $i, j$. If $\underline{x}^{i}=\underline{x}^{j}$ it follows by Step 2 that either $F^{i} \leq F^{j}$ or $F^{i} \geq F^{j}$ almost everywhere. Otherwise assume, without loss of generality, $\underline{x}^{j}<\underline{x}^{i}$. Thus, $0=w^{j}\left(\underline{x}^{j}\right) \leq w^{i}\left(\underline{x}^{j}\right)$ and hence, by Step $2, F^{i} \leq F^{j}$ almost everywhere. Hence, w.l.o.g., we assume for the rest of the proof $F^{i} \leq F^{j}$ almost everywhere. If $F^{i}<F^{j}$ on a set with positive Lebesgue measure it follows that

$$
\int_{\mathbb{R}_{+}}\left(1-F^{j}(x)\right) \phi^{\prime}(x) \mathrm{d} x<\int_{\mathbb{R}_{+}}\left(1-F^{i}(x)\right) \phi^{\prime}(x) \mathrm{d} x
$$

This violates the feasibility condition (Lemma 8). Thus $F^{i}=F^{j}$ almost everywhere. By continuity of $F^{i}$ and $F^{j}$, it follows that $F^{i}=F^{j}$.

Q.E.D.

Proof of Lemma 5: Define the payoff process as $Z_{t}=w\left(X_{t}\right)-\int_{0}^{t} c\left(X_{s}\right) \mathrm{d} s$ and the process $H$ by $H_{t}=\sup _{\tau} \mathbb{E}\left[Z_{\tau}\right]$. The process $H$ is a supermartingale (see for example Theorem 2.2 in Peskir and Shiryaev, 2006). By the Doob-Meyer decomposition theorem (Theorem 3.1 in Peskir and Shiryaev, 2006), there exists a unique martingale $\left(M_{t}\right)_{t \in \mathbb{R}_{+}}$ and a predictable, weakly decreasing process $\left(Q_{t}\right)_{t \in \mathbb{R}_{+}}, Q_{0}=0$ such that $H_{t}=M_{t}+Q_{t}$.

Let $D_{0}^{\prime}$ be the first time $Q_{t}$ decreases strictly, $D_{0}^{\prime}=\inf \left\{t: Q_{s}<Q_{t}\right.$ for $\left.s>t\right\}$. By Theorem 1.6 in Bismut and Skalli (1977), any stopping time $\tau$ which is optimal for the agent satisfies $\tau \leq D_{0}^{\prime}$.

By definition of $(\underline{x}, \bar{x})$ and the symmetry of the distributions $F$, each agent must continue at every point $x \in(\underline{x}, \bar{x})$ after some history. Due to the Markovian structure, it must be optimal for the agent not to stop whenever $X_{t} \in(\underline{x}, \bar{x})$. As it is optimal for the agent to continue at every point $x \in(\underline{x}, \bar{x})$ it follows that $Q_{t}=0$ for all $t<\tau_{(\underline{x}, \bar{x})}$. Hence, the process $\left(H_{t}\right)_{t \in\left[0, \tau_{(\underline{x}, \bar{x})}\right.}$ is a martingale.

As stopping is also optimal for all $x \in(\underline{x}, \bar{x})$ by the argument at the beginning of the proof of Lemma 3, it follows that $H_{t}=Z_{t}=w\left(X_{t}\right)-\int_{0}^{t} c\left(X_{s}\right) \mathrm{d} s$ for all $t \leq \tau_{(\underline{x}, \bar{x})}$.

As $\left(H_{t}\right)_{t \in\left[0, \tau_{(\underline{x}, \bar{x})}\right)}$ is a martingale, Doob's optional sampling theorem (see Peskir and 
Shiryaev, 2006, p.60) implies that for all $\underline{x}<a<x=X_{t}<b<\bar{x}$ and $t<\tau_{(\underline{x}, \bar{x})}$

$$
\begin{gathered}
w(x)-\int_{0}^{t} c\left(X_{s}\right) \mathrm{d} s=\mathbb{E}\left[w\left(X_{\tau_{(a, b)}}\right)-\int_{0}^{\tau_{(a, b)}} c\left(X_{s}\right) \mathrm{d} s \mid\left(X_{r}\right)_{r \leq t}\right] \\
\Rightarrow w(x)=\mathbb{P}\left[X_{\tau_{(a, b)}}=b \mid X_{t}=x\right](w(b)-w(a))+w(a) \\
-\mathbb{E}\left[\int_{t}^{\tau_{(a, b)}} c\left(X_{s}\right) \mathrm{d} s \mid X_{t}=x\right] .
\end{gathered}
$$

Let $q: \mathbb{R} \rightarrow \mathbb{R}$ be a solution to the ODE $\mu q^{\prime}(x)+\frac{\sigma^{2}}{2} q^{\prime \prime}(x)=c(x)$ for all $x \in(\underline{x}, \bar{x})$. By definition, $q$ is twice differentiable and applying Itô's Lemma gives that

$$
\begin{aligned}
q\left(X_{r}\right)-q\left(X_{t}\right) & =\int_{t}^{r} \mu q^{\prime}\left(X_{s}\right)+\frac{\sigma^{2}}{2} q^{\prime \prime}\left(X_{s}\right) \mathrm{d} s+\int_{t}^{r} \sigma q^{\prime}\left(X_{s}\right) \mathrm{d} B_{s} \\
& =\int_{t}^{r} c\left(X_{s}\right) \mathrm{d} s+\int_{t}^{r} \sigma q^{\prime}\left(X_{s}\right) \mathrm{d} B_{s} .
\end{aligned}
$$

Applying Doob's optional sampling theorem with the stopping time $\tau_{(a, b)}$ thus gives

$$
\mathbb{E}\left[q\left(X_{\tau_{(a, b)}}\right)-q\left(X_{t}\right) \mid X_{t}=x\right]=\mathbb{E}\left[\int_{t}^{\tau_{(a, b)}} c\left(X_{s}\right) \mathrm{d} s \mid X_{t}=x\right]
$$

Replacing the cost term in Eq. (4) yields

$$
w(x)=\mathbb{P}\left[X_{\tau_{(a, b)}}=b \mid X_{t}=x\right]([w(b)-q(b)]-[w(a)-q(a)])+w(a)+q(x) .
$$

It is known that that the probability is equal to (see, e.g., Beichelt, 2006, p.373, Example $7.5)$

$$
\mathbb{P}\left[X_{\tau_{(a, b)}}=b \mid X_{t}=x\right]=\frac{\exp \left(\frac{-2 \mu a}{\sigma^{2}}\right)-\exp \left(\frac{-2 \mu x}{\sigma^{2}}\right)}{\exp \left(\frac{-2 \mu a}{\sigma^{2}}\right)-\exp \left(\frac{-2 \mu b}{\sigma^{2}}\right)}
$$

and is thus twice differentiable for all $x \in(\underline{x}, \bar{x})$. Since $q$ is also twice differentiable in $x$ by definition, we find that $w$ is twice differentiable for all $x \in(\underline{x}, \bar{x})$. Applying Itô's Lemma to the process $\left(H_{t}\right)_{t \in[0, \tau(\underline{\underline{x}, \bar{x}})}$ gives that

$$
H_{t}=\int_{0}^{t} \mu w^{\prime}\left(X_{s}\right)+\frac{\sigma^{2}}{2} w^{\prime \prime}\left(X_{s}\right)-c\left(X_{s}\right) \mathrm{d} s+\int_{0}^{t} w^{\prime}\left(X_{s}\right) \sigma \mathrm{d} B_{s} .
$$

The first integral equals the predictable process $Q$ and the second part is the martingale $M$. As the process $\left(H_{t}\right)_{t \in\left[0, \tau_{(\underline{x}, \bar{x})}\right)}$ is a martingale, it follows that $Q \equiv 0$ and thus for all $x \in(\underline{x}, \bar{x})$

$$
c(x)=\mu w^{\prime}(x)+\frac{\sigma^{2}}{2} w^{\prime \prime}(x) .
$$


Proof of Lemma 6: By definition, $w(x)=0$, for all $x \leq \underline{x}$. Hence, the left derivative $\partial_{-} w(\underline{x})$ is zero. It remains to prove that the right derivative $\partial_{+} w(\underline{x})$ is also zero. Since $w(x)>0$ for all $x \in(\underline{x}, \bar{x})$, we know that $\partial_{+} w(\underline{x}) \geq 0$.

Towards a contradiction, assume $\partial_{+} w(\underline{x})>0$. For a given $w: \mathbb{R} \rightarrow \mathbb{R}_{+}$, let $\Psi: \mathbb{R} \rightarrow$ $\mathbb{R}$ be the unique function that satisfies the second order ordinary differential equation $c(x)=\mu \Psi^{\prime}(x)+\frac{\sigma^{2}}{2} \Psi^{\prime \prime}(x)$ with boundary conditions $\Psi(\underline{x})=0$ and $\Psi^{\prime}(\underline{x})=\partial_{+} w(\underline{x})$. As $\Psi^{\prime}(\underline{x})>0$, there exists a point $\hat{x}<\underline{x}$ such that $\Psi(x)<0=w(x)$ for all $x \in(\hat{x}, \underline{x})$. Consider the strategy $S=\min \left\{\tau_{\hat{x}, \bar{x}}, 1\right\}$ that stops when either the point $\hat{x}$ or $\bar{x}$ is reached or at time 1 . As $w(x)>\Psi(x)$ for all $x \in(\hat{x}, \underline{x})$, it follows that $\mathbb{E}\left(w\left(X_{S}\right)\right)>\mathbb{E}\left(\Psi\left(X_{S}\right)\right)$. Thus,

$$
\mathbb{E}\left(w\left(X_{S}\right)-\int_{0}^{S} c\left(X_{t}^{i}\right) \mathrm{d} t\right)>\mathbb{E}\left(\Psi\left(X_{S}\right)-\int_{0}^{S} c\left(X_{t}^{i}\right) \mathrm{d} t\right)
$$

Note that, by Itô's lemma, the process $\Psi\left(X_{t}^{i}\right)-\int_{0}^{t} c\left(X_{s}^{i}\right) \mathrm{d} s$ is a martingale. By Doob's optional sampling theorem (see Peskir and Shiryaev, 2006, p.60), agent $i$ is indifferent between the equilibrium strategy $\tau$ and the bounded time strategy $S$, i.e.,

$$
\mathbb{E}\left(\Psi\left(X_{S}\right)-\int_{0}^{S} c\left(X_{t}^{i}\right) \mathrm{d} t\right)=\mathbb{E}\left(\Psi\left(X_{\tau}\right)-\int_{0}^{\tau} c\left(X_{t}^{i}\right) \mathrm{d} t\right)=\mathbb{E}\left(w\left(X_{\tau}\right)-\int_{0}^{\tau} c\left(X_{t}^{i}\right) \mathrm{d} t\right)
$$

The last step follows because $w(x)$ and $\Psi(x)$ coincide for all $x \in(\underline{x}, \bar{x})$, since they solve the same differential equation, Eq. (1), with the same boundary conditions on the support (the only difference is that $\Psi(x)$ also satisfies Eq. (1) for $x \notin[\underline{x}, \bar{x}]$ ). Consequently, the strategy $S$ is a profitable deviation, which contradicts the equilibrium assumption. Q.E.D.

Proof of Lemma 7: By construction of $F, F(\underline{x})=0$. Clearly, $F$ is increasing on $(\underline{x}, \bar{x})$, as the derivative with respect to $x$,

$$
F^{\prime}(x)=\frac{F(x)^{2-n}}{(n-1)}\left(\frac{2}{\sigma^{2}} \int_{\underline{x}}^{x} c(z) \phi(x-z) \mathrm{d} z\right)
$$

is greater than zero for all $x>\underline{x}$. It remains to show that there exists an $x>\underline{x}$ such that $F(x)=1$. We can bound $F$ from below using a lower bound on the derivative of $F^{n-1}$ :

$$
\begin{aligned}
F(x)^{n-1} & =\frac{1}{\mu} \int_{\underline{x}}^{x} c(z)(1-\phi(x-z)) \mathrm{d} z \\
& \geq \frac{\underline{c}}{\mu}\left(x-\underline{x}-\frac{\sigma^{2}}{2 \mu}(1-\phi(x-\underline{x}))\right) \geq \frac{\underline{c}}{\mu}\left(x-\underline{x}-\frac{\sigma^{2}}{2 \mu}\right)
\end{aligned}
$$

By Assumption 1, $\underline{c}>0$. Continuity of $F$ implies that there exists a unique point $\bar{x}>\underline{x}$ such that $F(\bar{x})=1$.

Q.E.D. 
Proof of Lemma 8: Observe that, by Itô's lemma, $\left(\phi\left(X_{t}\right)\right)_{t \in \mathbb{R}_{+}}$is a martingale. Hence, by Doob's optional sampling theorem (see Peskir and Shiryaev, 2006, p.60), for any bounded stopping time $\tau$,

$$
1=\phi\left(X_{0}\right)=\mathbb{E}\left[\phi\left(X_{\tau}\right)\right]=\int_{\underline{x}}^{\bar{x}} \phi(x) H^{\prime}(x) \mathrm{d} x .
$$

Proof of Proposition 1: Uniqueness: As $F$ is absolutely continuous, the right endpoint $\bar{x}$ satisfies $(a): 1=\int_{\underline{x}}^{\bar{x}} F^{\prime}(x ; \underline{x}, \bar{x}) \mathrm{d} x$. By Lemma 7 , there exists a unique $\bar{x}$ for every $\underline{x}$ such that $(a)$ is satisfied. Lemma 8 states that any feasible distribution satisfies $(b): 1=\int_{\underline{x}}^{\bar{x}} F^{\prime}(x ; \underline{x}) \phi(x) \mathrm{d} x$. We show that intersection of the set of solutions to equation $(a)$ and to equation $(b)$ consists of a single point. Since $F^{\prime}(x ; \underline{x}, \bar{x})$ is independent of $\bar{x}$, we henceforth drop the dependency in our notation. By the implicit function theorem, the set of solutions to $(a)$ satisfies

$$
\frac{\partial \bar{x}}{\partial \underline{x}}=-\frac{-\overbrace{F^{\prime}(\underline{x} ; \underline{x})}^{=0}+\int_{\underline{x}}^{\bar{x}} \frac{\partial}{\partial \underline{x}} F^{\prime}(x ; \underline{x}) \mathrm{d} x}{F^{\prime}(\bar{x} ; \underline{x})}=-\frac{\int_{\underline{x}}^{\bar{x}} \frac{\partial}{\partial \underline{x}} F^{\prime}(x ; \underline{x}) \mathrm{d} x}{F^{\prime}(\bar{x} ; \underline{x})} .
$$

Applying the implicit function theorem to $(b)$ gives us

$$
\begin{aligned}
\frac{\partial \bar{x}}{\partial \underline{x}} & =-\frac{-\overbrace{F^{\prime}(\underline{x} ; \underline{x})}^{=0}+\int_{\underline{x}}^{\bar{x}} \frac{\partial}{\partial \underline{x}} F^{\prime}(x ; \underline{x}) \phi(x) \mathrm{d} x}{F^{\prime}(\bar{x} ; \underline{x}) \phi(\bar{x})}=-\frac{\int_{\underline{x}}^{\bar{x}} \frac{\partial}{\partial \underline{x}} F^{\prime}(x ; \underline{x}) \overbrace{\phi(x-\bar{x})}^{>1} \mathrm{~d} x}{F^{\prime}(\bar{x} ; \underline{x})} \\
& <-\frac{\int_{\underline{x}}^{\bar{x}} \frac{\partial}{\partial \underline{x}} F^{\prime}(x ; \underline{x}) \mathrm{d} x}{F^{\prime}(\bar{x} ; \underline{x})} .
\end{aligned}
$$

The last inequality follows from $\frac{\partial}{\partial \underline{x}} F^{\prime}(x ; \underline{x}) \geq 0$. As $(5)>(6)$, the solution sets to equation $(a)$ and $(b)$ intersect at most once. Thus, in equilibrium, both the left and the right endpoint are unique.

Existence: We have shown in Lemma 7 that, for every $\underline{x}$, there exists a unique $\bar{x}$ such that $F(\cdot ; \underline{x}, \bar{x})$ is a distribution function. Furthermore, rewriting the last inequality in the proof of Lemma 7 , we get $\bar{x} \leq \underline{x}+\frac{\mu}{\underline{c}}+\frac{\sigma^{2}}{2 \mu}$ and thus $\underline{x} \rightarrow-\infty \Rightarrow \bar{x} \rightarrow-\infty$. Consider a left endpoint $\underline{x} \geq 0$ and a right endpoint $\bar{x}$ such that $(a)$ is satisfied. Then, $\phi(x)<1$ for all $x \in[\underline{x}, \bar{x}]$ and hence $\mathbb{E}(\phi(x))<1$ for $x \sim F(\cdot, \underline{x}, \bar{x})$. Now consider a left endpoint $\underline{x}<0$ and a right endpoint $\bar{x}<0$ such that $(a)$ is satisfied. Then, $\phi(x)>1$ for all $x \in[\underline{x}, \bar{x}]$ and hence $\mathbb{E}(\phi(x))>1$ for $x \sim F(\cdot, \underline{x}, \bar{x})$. Equation $(b)$ is equivalent to $\mathbb{E}(\phi(x))=1$. By continuity and the Intermediate Value Theorem, there exists an $\underline{x}, \bar{x}$ such $(a)$ and $(b)$ are satisfied. This concludes the first implication of the statement in the proposition.

It remains to show that if all players play according to the distribution $F$, there is 
no profitable deviation. For this purpose, define $\Psi$ as the unique solution to Eq. (1) with the boundary conditions $\Psi(\underline{x})=0$ and $\Psi^{\prime}(\underline{x})=0$. By construction, the process $\Psi\left(X_{t}^{i}\right)-\int_{0}^{t} c\left(X_{s}^{i}\right) \mathrm{d} s$ is a martingale and $\Psi(x)=w(x)$ for all $x \in[\underline{x}, \bar{x}]$. As $\Psi^{\prime}(x)<0$ for $x<\underline{x}$ and $\Psi^{\prime}(x)>0$ for $x>\bar{x}, \Psi(x)>w(x)$ for all $x \notin[\underline{x}, \bar{x}]$. For every stopping time $S$, we use Itô's Lemma to calculate the expected value

$$
\begin{aligned}
\mathbb{E}\left(w\left(X_{S}\right)-\int_{0}^{S} c\left(X_{t}\right) \mathrm{d} t\right) & \leq \mathbb{E}\left(\Psi\left(X_{S}\right)-\int_{0}^{S} c\left(X_{t}\right) \mathrm{d} t\right) \\
& =\Psi\left(X_{0}\right)=w\left(X_{0}\right)=\mathbb{E}\left(w\left(X_{\tau}\right)\right) .
\end{aligned}
$$

The last equality results, as each agent is indifferent to stop immediately with the expected payoff $w\left(X_{0}\right)$ or to play the equilibrium strategy with the expected payoff $\mathbb{E}\left(w\left(X_{\tau}\right)\right)$. The inequality implies that any possible deviation strategy obtains a weakly lower payoff than the equilibrium candidate.

Q.E.D.

Proof of Proposition 2: The function $\Phi$ is Lipschitz continuous with constant $\frac{1}{\sqrt{2 \pi}}$. Consequently, it suffices to prove Lipschitz continuity of $F^{-1}$ to get the Lipschitz continuity of $F^{-1} \circ \Phi$. The density $f$ is

$$
f(x)=\frac{F(x)^{-n+2}}{n-1} \frac{2}{\sigma^{2}} \int_{\underline{x}}^{x} c(z) \phi(x-z) \mathrm{d} z=\frac{F(x)^{-n+2}}{n-1} \frac{2}{\sigma^{2}}\left(\int_{\underline{x}}^{x} c(z) \mathrm{d} z-\mu F(x)^{n-1}\right) .
$$

As $f(x)>0$ for all $x>\underline{x}$, it suffices to show Lipschitz continuity of $F^{-1}$ at 0 . We substitute $x=F^{-1}(y)$ to get

$$
\left(f \circ F^{-1}\right)(y) \geq \frac{1}{n-1} \frac{2}{\sigma^{2}}\left(y^{2-n}\left(\min _{z \in[\underline{x}, \bar{x}]} c(z)\right)\left(F^{-1}(y)-F^{-1}(0)\right)-\mu y\right) .
$$

We define $\underline{c}=\inf _{z \in[\underline{x}, \bar{x}]} c(z)$. Rearranging with respect to $F^{-1}(y)-F^{-1}(0)$ gives

$$
F^{-1}(y)-F^{-1}(0) \leq\left(\frac{(n-1) \sigma^{2}}{2}\left(f \circ F^{-1}\right)(y)+\mu y\right) \frac{y^{n-2}}{\underline{c}} \leq\left(\frac{(n-1) \sigma^{2}}{2} f(\bar{x})+\mu\right) \frac{y^{n-2}}{\underline{c}} .
$$

This proves the Lipschitz continuity of $F^{-1}$ for $n>2$. Note that for two agents $n=2$ the function $F^{-1}$ is not Lipschitz continuous as $f(\underline{x})=0$. However, we show in the following paragraph that $F^{-1} \circ \Phi$ is Lipschitz continuous for $n=2$.

$$
F(x)=\int_{\underline{x}}^{x} \frac{c(z)}{\mu}\left(1-\phi(x-z) \mathrm{d} z \leq\left(\sup _{z \in[\underline{x}, \bar{x}]} \frac{c(z)}{\mu}\right)\left(x-\underline{x}-\frac{\sigma^{2}}{2 \mu}(1-\phi(x-\underline{x}))\right)\right.
$$

We define $\bar{c}=\sup _{z \in[\underline{x}, \bar{x}]} c(z)$. A second order Taylor expansion around $\underline{x}$ yields that, for an open ball around $\underline{x}$ and $\underline{x}<x$, we have the following upper bound

$$
x-\underline{x}-\frac{\sigma^{2}}{2 \mu}(1-\phi(x-\underline{x})) \leq \frac{2 \mu}{\sigma^{2}}(1-\phi(x-\underline{x}))^{2} .
$$


For an open ball around $\underline{x}$, we get an upper bound on $F(x) \leq \frac{2 \bar{c}}{\sigma^{2}}(1-\phi(x-\underline{x}))^{2}$ and hence the following estimate

$$
1-\phi(x-\underline{x}) \geq \sqrt{\frac{\sigma^{2}}{2 \bar{c}} F(x)} .
$$

We use this estimate to obtain a lower bound on $f$ depending only on $F$

$$
f(x)=\frac{2}{\sigma^{2}}\left(\int_{\underline{x}}^{x} c(z) \phi(x-z) \mathrm{d} z\right) \geq \frac{2 \underline{c}}{\sigma^{2}}\left(\frac{\sigma^{2}}{2 \mu}(1-\phi(x-\underline{x}))\right) \geq \frac{\underline{c}}{\mu} \sqrt{\frac{\sigma^{2}}{2 \bar{c}} F(x)} .
$$

Consequently, there exists an $\epsilon>\underline{x}$ such that, for all $x \in[\underline{x}, \epsilon)$, we have an upper bound on $\frac{\left(\phi \circ \Phi^{-1} \circ F\right)(x)}{f(x)}$. Taking the limit $x \rightarrow \underline{x}$ yields

$$
\lim _{x \rightarrow \underline{x}} \frac{\left(\phi \circ \Phi^{-1} \circ F\right)(x)}{f(x)} \leq \lim _{x \rightarrow \underline{x}} \frac{\left(\phi \circ \Phi^{-1} \circ F\right)(x)}{\frac{c}{\mu} \sqrt{\frac{\sigma^{2}}{2 \bar{c}} F(x)}} \leq \sqrt{\frac{2 \bar{c} \mu^{2}}{\underline{c}^{2} \sigma^{2}}} \lim _{y \rightarrow 0} \frac{\left(\phi \circ \Phi^{-1}\right)(y)}{\sqrt{y}}=0 .
$$

Hence, we have shown that $F^{-1} \circ \Phi$ is Lipschitz continuous. Thus, there exists a bounded time stopping strategy (for an explicit construction, see Ankirchner and Strack, 2011, Theorem 2) which embeds $F$.

Q.E.D.

Proof of Proposition 3: The stopping strategy constructed in Ankirchner and Strack (2011) is not a barrier stopping time. However, Rost (1976) shows in his Theorem 1 that if there exists a stopping time that embeds the distribution $F$, there also exists a barrier stopping time which embeds F. Furthermore, by Theorem 2 in Rost (1976), this first leaving time of a set minimizes

$$
\mathbb{E}(\tau-\min \{\tau, t\})=\mathbb{P}(\tau \geq t) \mathbb{E}(\tau-t \mid \tau \geq t)
$$

over the set of all stopping times that embed $F$ for all $t \in \mathbb{R}_{+}$. As the stopping time constructed in Ankirchner and Strack (2011) is bounded by $T$, the first leaving time of a set constructed in Rost (1976) is also bounded by $T$.

Q.E.D.

Proof of Lemma 9: For any bounded stopping time, for any $\sigma>0$, feasibility implies that $\underline{x} \leq 0$. By contradiction, assume there exists a constant $\epsilon$ such that $\underline{x} \leq \epsilon<0$ for some sequence $\left(\sigma_{k}\right)_{k \in \mathbb{N}}$ with $\lim _{k \rightarrow \infty} \sigma_{k}=0$. Then $F^{\prime}$ is bounded away from zero by

$$
\begin{aligned}
F^{\prime}(x) & =\frac{F(x)^{2-n}}{n-1} \frac{2}{\sigma^{2}} \int_{\underline{x}}^{x} c(z) \phi(x-z) \mathrm{d} z \geq \frac{1}{n-1} \frac{2}{\sigma^{2}} \int_{\epsilon}^{x} c(z) \phi(x-z) \mathrm{d} z \\
& \geq \frac{\underline{c}}{\mu(n-1)}(1-\phi(x-\epsilon)) .
\end{aligned}
$$


For every point $x<0, \lim _{\sigma_{k} \rightarrow 0} \phi(x)=\infty$. Thus,

$$
\lim _{\sigma_{k} \rightarrow 0} \int_{\underline{x}}^{0} F^{\prime}(x) \phi(x) \mathrm{d} x>1
$$

which contradicts feasibility, because

$$
\int_{\underline{x}}^{0} F^{\prime}(x) \phi(x) \mathrm{d} x \leq \int_{\underline{x}}^{\bar{x}} F^{\prime}(x) \phi(x) \mathrm{d} x=1 .
$$

Proof of Proposition 5: In equilibrium, agents are indifferent between stopping immediately and the equilibrium strategy. Their expected profit is thus $w(0)$, which is strictly positive as $\underline{x}<0$.

Proof of Lemma 10 And Lemma 11: Define $\Delta=\bar{x}-\underline{x}$. Rearranging the density condition $1=F(\bar{x})=\frac{c}{\mu}\left[\Delta-\frac{\sigma^{2}}{2 \mu}(1-\phi(\Delta))\right]$ yields

$$
\exp \left(\frac{-2 \mu \Delta}{\sigma^{2}}\right)=-\frac{2 \mu}{\sigma^{2}}\left[\Delta-\left(\frac{\mu}{c}+\frac{\sigma^{2}}{2 \mu}\right)\right] \text {. }
$$

The solution to the transcendental algebraic equation $e^{-a \Delta}=b(\Delta-d)$ is $\Delta=d+$ $\frac{1}{a} W_{0}\left(\frac{a e^{-a d}}{b}\right)$, where $W_{0}:\left[-\frac{1}{e}, \infty\right) \rightarrow \mathbb{R}_{+}$is the principal branch of the Lambert $W$ function. This branch is implicitly defined on $\left[-\frac{1}{e}, \infty\right)$ as the unique solution of $x=$ $W(x) \exp (W(x)), W \geq-1$. Hence,

$$
\Delta=\frac{\mu}{c}+\frac{\sigma^{2}}{2 \mu}\left[1+W_{0}\left(-\exp \left(-1-\frac{2 \mu^{2}}{c \sigma^{2}}\right)\right)\right]
$$

and

$$
\begin{aligned}
\phi(\Delta) & =\exp \left(\frac{-2 \mu^{2}}{c \sigma^{2}}-1-W_{0}\left(-\exp \left(-1-\frac{2 \mu^{2}}{c \sigma^{2}}\right)\right)\right) \\
& =\exp \left(-1-y-W_{0}(-\exp (-1-y))\right)=h(y) .
\end{aligned}
$$

Note that $\phi(\Delta)$ only depends on $y=\frac{2 \mu^{2}}{c \sigma^{2}}$. As $\Delta \geq 0$ it follows that $h(y)=\phi(\Delta) \in[0,1]$. Moreover, $h: \mathbb{R}_{+} \rightarrow[0,1]$ is strictly decreasing in $y$, as $W_{0}$ and exp are strictly increasing functions. For constant costs, the feasibility condition from Lemma 8 reduces to

$$
1=\int_{\underline{x}}^{\bar{x}} F^{\prime}(x) \phi(x) \mathrm{d} x=\frac{c \sigma^{2}}{2 \mu^{2}}\left[\frac{1}{2} \phi(\underline{x})+\frac{1}{2} \phi(2 \bar{x}-\underline{x})-\phi(\bar{x})\right] .
$$

Dividing by $\phi(\underline{x})$ gives

$$
\begin{aligned}
\phi(-\underline{x}) & =\frac{c \sigma^{2}}{2 \mu^{2}}\left[\frac{1}{2}+\frac{1}{2} \phi(\Delta)^{2}-\phi(\Delta)\right]=\frac{1}{y}\left[\frac{1}{2}+\frac{1}{2} h(y)^{2}-h(y)\right] \\
& =\frac{1}{2 y}(1-h(y))^{2}=g(y)
\end{aligned}
$$


As $\underline{x} \leq 0$ it follows that $g(y)=\phi(-\underline{x}) \in[0,1]$. The derivative of $g$ is given by

$$
g^{\prime}(y)=-\frac{1}{2 y^{2}}(1-h(y))^{2}+\frac{2}{2 y}(1-h(y)) h^{\prime}(y) .
$$

As $h$ is a strictly decreasing function from $\mathbb{R}_{+}$to $[0,1]$ it follows that $g: \mathbb{R}_{+} \rightarrow[0,1]$ is strictly decreasing in $y$. We calculate $\underline{x}$ as

$$
\begin{aligned}
\underline{x} & =-\phi^{-1}(\phi(-\underline{x}))=\frac{\sigma^{2}}{2 \mu} \log (g(y))=\frac{\sigma^{2}}{2 \mu} \log \left(\frac{1}{2 y}(1-h(y))^{2}\right) \\
& =-\frac{\sigma^{2}}{2 \mu}[2 \log (1-h(y))-\log (2 y)] .
\end{aligned}
$$

Simple algebraic transformations yield the expression for $\bar{x}$ in Lemma 10. We plug in $\underline{x}=\frac{\sigma^{2}}{2 \mu} \log (g(y))$ and $\phi(-\underline{x})=g(y)$ in the distribution for the constant cost case to get:

$$
\begin{aligned}
F(0) & =w(0)=\frac{c}{\mu}\left[-\underline{x}-\frac{\sigma^{2}}{2 \mu}(1-\phi(-\underline{x}))\right]=\frac{c}{\mu}\left[-\frac{\sigma^{2}}{2 \mu} \log (g(y))-\frac{\sigma^{2}}{2 \mu}(1-g(y))\right] \\
& =\frac{1}{y}[g(y)-\log (g(y))-1] .
\end{aligned}
$$

Hence, the value of $F(0)$ depends on the value of the fraction $y=\frac{2 \mu^{2}}{c \sigma^{2}}$ in the above way, which completes the proof of Lemma 11.

Q.E.D.

Proof of Proposition 6: By Lemma 11, it suffices to show that the profit $\pi=$ $w\left(X_{0}\right)=w(0)=F(0)$ is increasing in $y$. Consider the following expression from the previous proof:

$$
F(0)=\frac{1}{y}[g(y)-\log (g(y))-1]
$$

Note $\frac{1}{y}$ is positive and $g(y)-\log (g(y))-1$ is positive as $F(0) \geq 0$. The function $x-\log (x)$ is increasing in $x$. Hence, $g(y)-\log (g(y))-1$ is decreasing in $y$, because $g(y)$ is decreasing in $y$. As $\frac{1}{y}$ is also decreasing in $y$, the product $\frac{1}{y}[g(y)-\log (g(y))-1]$ of two decreasing positive functions is decreasing in $y$.

Q.E.D.

\section{ACKNOWLEDGEMENTS}

We are indebted to Dirk Bergemann, Benny Moldovanu, Sven Rady, and Tymon Tatur for their enduring support of this work. Furthermore, we would like to thank Mehmet Ekmekci, Eduardo Faingold, Paul Heidhues, Johannes Hörner, Philippe Jehiel, Navin Kartik, Sebastian Kranz, Giuseppe Moscarini, Ron Siegel, seminar audiences in Bonn, Cologne, Frankfurt, Maastricht, Mannheim, Yale, the Workshop on Stochastic Methods in Game Theory 2010 in Erice, the World Congress of the Econometric Society 2010 in Shanghai, the Stony Brook Game Theory Festival 2011, the European Winter Meeting 
of the Econometric Society 2012 in Konstanz, and the Meeting of the Society for the Advancement of Economic Theory 2013 in Paris for helpful suggestions. Philipp Strack is particularly grateful for an extended stay at Yale, during which a large part of this research was conducted. Financial support by Bonn Graduate School of Economics, Deutsche Forschungsgemeinschaft, Hausdorff Center for Mathematics, and SFB TR 15 is gratefully acknowledged.

\section{REFERENCES}

Amann, E. and Leininger, W. (1996). Asymmetric all-pay auctions with incomplete information: The two-player case. Games and Economic Behavior, 14:1-18.

Ankirchner, S. and Strack, P. (2011). Skorokhod embeddings in bounded time. Stochastics and Dynamics, $11: 215-226$.

Aumann, R. J. (1961). Mixed and behavior strategies in infinite extensive games. Technical report, DTIC Document.

Baye, M., Kovenock, D., and de Vries, C. (1996). The all-pay auction with complete information. Economic Theory, 8:291-305.

Beichelt, F. (2006). Stochastic Processes in Science, Engineering and Finance. Chapman \& Hall CRC, Taylor \& Francis Group.

Bismut, J.-M. (1979). Temps d'arrêt optimal, quasi-temps d'arrêt et retournement du temps. The Annals of Probability, pages 933-964.

Bismut, J.-M. and Skalli, B. (1977). Temps d'arrêt optimal, théorie générale des processus et processus de markov. Probability Theory and Related Fields, 39:301-313.

Burdett, K. and Judd, K. L. (1983). Equilibrium price dispersion. Econometrica, 51:955-969.

Feng, H. and Hobson, D. (2014a). Gambling in contests with random initial law. Annals of Applied Probability, forthcoming.

Feng, H. and Hobson, D. (2014b). Gambling in contests with regret. Mathematical Finance, forthcoming.

Harris, C. and Vickers, J. (1987). Racing with uncertainty. Review of Economic Studies, 54:1-21.

Hillman, A. and Riley, J. (1989). Politically contestable rents and transfers. Economics and Politics, $1: 17-39$

Hillman, A. and Samet, D. (1987). Dissipation of contestable rents by small numbers of contenders. Public Choice, 54:63-82.

Hopenhayn, H. and Squintani, F. (2011). Preemption games with private information. Review of Economic Studies, 78:667-692.

Karlin, S. (1953). Reduction of certain classes of games to integral equations. in: H. Kuhn \& A. Tucker (Eds.), 'Contributions to the Theory of Games, II.', Vol.28 of Annals of Mathematical Studies, Princeton University Press, Princeton, pp.125-128.

Krishna, V. and Morgan, J. (1997). An analysis of the war of attrition and the all-pay auction. Journal of Economic Theory, 72:343-362.

Laraki, R. and Solan, E. (2013). Equilibrium in two-player non-zero-sum dynkin games in continuous time. Stochastics, 85:997-1014.

Laraki, R., Solan, E., and Vieille, N. (2005). Continuous-time games of timing. Journal of Economic Theory, 120:206-238.

Lazear, E. P. and Rosen, S. (1981). Rank-order tournaments as optimal labor contracts. Journal of Political Economy, 89:841-864. 
Moldovanu, B. and Sela, A. (2001). The optimal allocation of prizes in contests. American Economic Review, 91:542-558.

Moscarini, G. and Smith, L. (2007). Optimal dynamic contests. Working Paper.

Oblój, J. (2004). The Skorokhod embedding problem and its offspring. Probability Surveys, 1:321-392.

Park, A. and Smith, L. (2008). Caller number five and related timing games. Theoretical Economics, $3: 231-256$.

Peskir, G. and Shiryaev, A. (2006). Optimal Stopping and Free-Boundary Problems. Birkhäuser Verlag, Basel, Boston, Berlin.

Rost, H. (1976). Skorokhod stopping times of minimal variance. Séminaire de Probabilités (Strasbourg), 10:194-208.

Seel, C. and Strack, P. (2013). Gambling in contests. Journal of Economic Theory, 148:2033-2048.

Shmaya, E. and Solan, E. (2014). Equivalence between random stopping times in continuous time. arXiv preprint arXiv:1403.7886.

Siegel, R. (2009). All-pay contests. Econometrica, 77:71-92.

Siegel, R. (2010). Asymmetric contests with conditional investments. American Economic Review, 100:2230-2260.

Skorokhod, A. V. (1961). Issledovaniya po teorii sluchainykh protsessov (stokhasticheskie differentsialnye uravneniya i predelnye teoremy dlya protsessov markova). Izdat Kiev University.

Skorokhod, A. V. (1965). Studies in the theory of random processes. Translated from the Russian by Scripta Technica, Inc. Addison-Wesley Publishing Co., Inc., Reading, Mass.

Solan, E., Tsirelson, B., and Vieille, N. (2012). Random stopping times in stopping problems and stopping games. arXiv preprint arXiv:1211.5802.

Touzi, N. and Vieille, N. (2002). Continuous-time dynkin games with mixed strategies. SIAM Journal on Control and Optimization, 41(4):1073-1088.

Tullock, G. (1980). Efficient rent seeking, in: J.Buchanan, R.Tollison and G.Tullock, (eds.), Towards a Theory of Rent-Seeking Society. Texas A\&M University Press. 